\section{Impacto da orientação para a prática regular de atividade física dois anos após o parto}

\section{Impact of guidance on the practice of regular physical activity two years after giving birth}

Simone Cristina Scarpa 1

Carolina Harumi Kurashima 2

Monica Yuri Takito 3

\begin{abstract}
Objectives:to evaluate changes in the practice of physical activity (PA) and the barriers to adopting a healthy lifestyle among women who have received guidance on nutrition and PA during pregnancy and in the first year after giving birth.

Methods: cohort study with 57 women (Control Group $=29$ and Intervention Group $=28$ ), and mean age $28( \pm 6)$ years. The information was obtained through home visit interviews (during intervention) and telephone interviews (one year after the end of intervention), using the PA questionnaire. To analyze the barriers a semi-structured interview with open questions was carried out, these being codified and grouped for data analysis. Chi-square, Mann-Whitney $U$ and Friedman tests were carried out.

Results: at six months, only $30 \%$ of the women in the Intervention Group were practicing leisure-time PA at least 120 minutes per week, as opposed to 10\% from the Control Group, decreasing to $18 \%$ and $4 \%$ respectively two years after giving birth. The principal barriers to practicing $P A$ regularly were: lack of time (44\%), taking care of children (37\%), work (21\%), domestic chores (21\%) and laziness (26\%).

Conclusions: future programs should prioritize the acquisition of knowledge, with a view to adopting an active lifestyle after giving birth, considering the maternal barriers.
\end{abstract}

Key words Pregnant women, Postpartum, Motor activity, Barriers, Motivation
1-3 Escola de Educação Física e Esporte. Universidade de São Paulo. Av. Prof. Mello de Morais, 65. Cidade Universitária. São Paulo, SP, Brasil. CEP: 05.508-030. E-mail: simone.scarpa@usp.br

\section{Resumo}

Objetivos: avaliar as modificações na prática de atividade física $(A F)$ e as barreiras para adoção de um estilo de vida saudável em mulheres que receberam orientações nutricionais e de AF durante a gestação e no primeiro ano após o parto.

Métodos: estudo de coorte com 57 mulheres $($ Grupo Controle $=29$ e Grupo Intervenção $=28), e$ idade média igual a $28( \pm 6)$ anos. As informações foram obtidas através de entrevistas em visita domiciliar (durante a intervenção) e inquérito telefônico (um ano após o término da intervenção), utilizou-se questionário de AF. Para análise das barreiras foi realizada uma entrevista semiestruturada com questões abertas, as quais foram codificadas e agrupadas para análise dos dados. Foram realizados testes de qui-quadrado, Mann-Whitney U e Friedman.

Resultados: aos seis meses, apenas 30\% das mulheres do grupo Intervenção realizavam AF no lazer pelo menos 120 minutos por semana, contra $10 \%$ do grupo Controle, reduzindo para $18 \%$ e $4 \%$, respectivamente, dois anos após o parto. As principais barreiras à prática de AF regular foram: falta de tempo (44\%), cuidado com os filhos (37\%), trabalho (21\%), afazeres domésticos (21\%) e comodismo $(26 \%)$.

Conclusões: futuros programas devem priorizar a aquisição de conhecimento, visando à adoção de um estilo de vida ativo no pós-parto, considerando as barreiras maternas.

Palavras-chave Gestantes, Pós-parto, Atividade física, Barreira, Motivação 


\section{Introdução}

A estratégia global ${ }^{1}$ para alimentação, atividade física e saúde preconiza que a adoção de hábitos alimentares saudáveis e a prática de atividade física regular apresentam efeito protetor para diversas patologias. A adoção de um estilo de vida saudável tem sido altamente recomendada para a população, sendo a atividade física um foco de atenção na promoção da saúde. Para tal, diversos órgãos de saúde internacionais, como o Centro de Controle e Prevenção de Doenças (CDC), o Colégio Americano de Medicina Esportiva (ACSM) e a Organização Mundial da Saúde (OMS), assim como a Secretaria de Saúde do Estado de São Paulo (Agita São Paulo CELAFISCS) têm recomendado a realização de pelo menos 30 minutos de atividade física moderada, de forma contínua ou acumulada em pelo menos cinco dias da semana. $2-4$

Tais mudanças de comportamento com vista a adoção de estilo de vida ativo também tem sido preconizadas às mulheres no período gestacional e posterior ao parto, destacando-se como fator de proteção à saúde materna e fetal.5,6 Dentre os benefícios merecem destaque o adequado ganho de peso gestacional e a menor retenção de peso pós-parto. ${ }^{5}$ Considerando o reduzido controle sobre fatores genéticos e biológicos, o padrão de atividade física e a alimentação são fatores potencialmente modificáveis refreando a influência dos fatores sobre os quais temos pouco ou nenhum controle. 7 Os períodos gestacionais e pós-parto modificam o estilo de vida da mulher e representam um momento oportuno para a promoção da atividade física e alimentação saudável visando a benefícios futuros à saúde de ambos (binômio mãe-bebê). 8,9

A realização de atividade física no puerpério deve ser iniciada aos poucos e aumentada de forma gradual, especialmente nas primeiras seis semanas pós-parto. Após esse período, a mulher estará apta a seguir as recomendações de atividade física para a população em geral.10 Estudos de intervenção promovendo a prática da atividade física tem obtido pouco sucesso, ${ }^{11,12}$ visto que a manutenção da mesma sofre redução com o tempo, indicando a necessidade de melhor compreensão do seu significado por parte dessas mulheres e da capacidade de superar barreiras e obstáculos. ${ }^{13}$

Desse modo, este estudo tem por objetivo avaliar a prática regular de atividade física e identificar as barreiras relatadas para a manutenção de um estilo de vida ativo em mulheres que receberam orientações nutricionais e de atividade física durante a gestação e no primeiro ano após o parto.

\section{Métodos}

Trata-se de um ensaio clínico não randomizado oriundo de duas coortes de mulheres saudáveis, de 18 a 40 anos e com nascimentos únicos, captadas durante a gestação. Foram critérios de exclusão: duração da gestação inferior a 37 semanas, patologia que restringisse a prática de exercício físico e gestação dois anos após o parto.

No período de maio/2005 a julho/2006, todas as gestantes que compareceram às Unidades Básicas de Saúde do subdistrito do Butantã do município de São Paulo foram convidadas a participar da primeira parte do estudo. O grupo Controle foi captado no primeiro ano e o grupo Intervenção após o término da coorte histórica de Controle, para minimizar possível viés de contaminação da amostra visto que as mulheres utilizavam o mesmo serviço público de pré-natal. Esses grupos foram acompanhados durante a gestação até os doze meses após o parto, sendo que o grupo Controle recebeu o atendimento padrão do serviço, não tendo contato com nutricionistas que realizavam a intervenção. Para evitar viés de informação, a intervenção não era realizada pelos entrevistadores e, sim, por nutricionistas que realizavam exclusivamente as orientações.

Após um ano sem contato (dois anos após o parto), foram contatadas 40 mulheres do grupo Controle e 38 mulheres do grupo Intervenção por telefone. Foram excluídas três mulheres que estavam grávidas novamente, sete se recusaram a permanecer no estudo e dez solicitaram retorno da ligação em outro horário, mas não foi mais possível o contato. Resultando em amostra de 28 mulheres no grupo Intervenção e de 29 no grupo Controle. A mudança com grande facilidade de linha telefônica, em especial aparelhos celulares pré-pagos impossibilitou a localização das mulheres. Adicionalmente, a dificuldade em responder as questões por telefone com crianças pequenas fez com que grande número de mulheres solicitasse retorno da ligação em outro horário ou se recusassem a responder. Para o desenvolvimento do protocolo de intervenção no estilo de vida, utilizaram-se as recomendações nutricionais de alimentação saudável para gestantes e puérperas do Institute of Medicine14 e do American College of Obstetricians and Gynecology6 e promoção de atividade física para a população. ${ }^{4}$ As orientações foram fornecidas individualmente às mulheres do grupo Intervenção através de contato telefônico por nutricionistas previamente treinadas. Em relação à atividade física, houve um incentivo à prática de caminhada, sendo esta preconizada por pelo menos cinco vezes na semana e com duração mínima de 30 
minutos. Foi enfatizada também a manutenção de um padrão de alimentação saudável e o consumo adequado de calorias. Após seis semanas pós-parto, as mulheres foram orientadas a retomar o padrão de atividade física praticado durante a gestação e buscar soluções para a realização de atividade física neste período em que o bebê necessita constantemente de cuidado. Posteriormente, contatos telefônicos foram feitos no $4^{\circ}, 7^{\circ}$ e $10^{\circ}$ mês após o parto para a realização de novas intervenções relacionadas a uma alimentação saudável e à retomada da prática de atividade física. As informações foram transmitidas de forma oral e através do envio de folhetos pelo correio, com conteúdo adequado para cada indivíduo, a fim de estimular a mudança de comportamento.

A coleta de dados foi realizada por entrevistadores treinados que preencheram questionários padronizados. A mensuração da atividade física foi realizada com questionário semiquantitativo validado de Chasan-Taber et al.,15 com a finalidade de estimar o tempo diário ou semanal despendido em 32 atividades, incluindo atividades domésticas e de cuidado (13 questões), ocupacionais (5 questões), esportes ou exercício ( 8 atividades), transporte/locomoção (3 questões) e inatividade (3 questões). A tradução e adequação das questões foram analisadas por três pesquisadores e um expert na língua inglesa. Os questionários foram aplicados em três momentos após o parto: $6^{\circ}$ mês, um e dois anos. A fim de padronizar a entrevista, as perguntas e todas as alternativas de resposta eram lidas pelos entrevistadores, em caso de dúvida ou demora na resposta repetia-se a questão. As mulheres eram solicitadas as atividades de uma semana típica dos meses anteriores.

O tempo gasto em cada atividade foi multiplicado pelo seu equivalente metabólico (MET), segundo o compêndio de atividades físicas, 16 para estimar a média semanal do gasto energético (METh.sem-1) para cada atividade. Todas as atividades físicas foram somadas para obter a estimativa do gasto médio semanal total. Além disso, o gasto médio semanal das atividades foi agrupado, segundo o tipo de atividade (doméstica, ocupacional, lazer, e locomoção). 15

O segundo momento foi constituído por questões abertas, com caráter qualitativo, com as quais foram identificadas as principais barreiras e motivações quanto à prática de atividade física. As últimas questões da entrevista semiestruturada com questões abertas foram elaboradas pensando na monitorização das recomendações, ou seja, identificar quais as recomendações foram mais relevantes para as mulheres da coorte de Intervenção e quais as recomendações que o grupo Controle recebeu por parte de profissionais da saúde após o parto até o dia da entrevista.

Adicionalmente foram obtidas informações para caracterização da amostra, tais como nível socioeconômico (escolaridade e renda), história obstétrica (paridade), rede de apoio (presença de companheiro, ajuda para cuidar do filho/casa), variáveis biológicas (idade, cor da pele, estado nutricional pré-gestacional) e comportamentais (tabagismo). As variáveis antropométricas foram as medidas da massa corporal e estatura recomendadas por Jellife e Jellife. ${ }^{17}$ Para a mensuração da massa corporal, utilizou-se uma balança Tanita ${ }^{\circledR}$ portátil digital (modelo 2001W-B), com acuidade de 100 gramas. A estatura foi mensurada por um estadiômetro portátil, com acuidade de um centímetro, no início da gestação. A padronização e controle de qualidade das medidas antropométricas foram executados mediante técnica recomendada por Habicht. 18 A partir do peso e estatura foi calculado o índice de massa corporal (IMC) (peso/estatura 2 ), e o estado nutricional no início da gestação classificado segundo Atalah et al. 19

A análise estatística inicialmente descreveu as coortes de Intervenção e Controle (medidas de tendência central e dispersão) quanto às características da amostra através do qui-quadrado, com correção de Yates, e do teste de diferença de médias para amostras independentes " $t$ " de Student. As variáveis de atividade física não seguem a normalidade, assim foi realizada estatística não paramétrica comparando o padrão de atividade física entre os dois grupos nos três momentos através do teste $\mathrm{U}$ de Mann-Whitney e, posteriormente, entre os momentos para cada grupo, através do teste de Friedman para amostras dependentes. Os dados foram digitados duplamente em banco construído com o programa Epi Info 6.0. Adotou-se nível de significância de 5\%. As análises foram realizadas utilizando-se o recurso estatístico SPSS for Windows versão 15.0 .

Este estudo foi aprovado pelo Comitê de Ética em Pesquisa local (2008/16). A pesquisa foi de baixo risco, sendo que os objetivos do estudo, bem como os procedimentos utilizados foram explicados oralmente às gestantes, em linguagem compreensível. Foi garantida a liberdade da gestante de recusar-se a participar ou de retirar seu consentimento em qualquer fase da pesquisa. As mulheres assinaram o termo de consentimento livre e esclarecido no momento do ingresso na coorte durante a gestação. Para o prosseguimento, cada mulher consentiu, oralmente, em continuar participando da pesquisa após as explicações. 


\section{Resultados}

A amostra foi composta por adultas jovens de baixo nível sócio-econômico. Não houve diferença significativa entre os grupos como pode ser observado nas Tabelas 1 e 2.

Quando questionadas sobre a amamentação, apenas dois quintos das mulheres de ambas as coortes afirmaram amamentar até os seis meses. Em relação à rede de apoio social, identificou-se que a maioria (90\%) das mulheres morava com o companheiro, mas somente $32,8 \%$ delas possuíam alguém para dividir as tarefas domésticas. Em relação aos cuidados com o bebê (vestir, dar banho, brincar, entre outras coisas), $60,7 \%$ delas possuíam alguém para auxiliá-las. Durante a gestação, $51,7 \%$ das mulheres trabalhavam. Um ano após, esse número de mulheres se manteve e após dois anos $55,2 \%$ da amostra total trabalhava (dados não apresentados). A ocupação predominante estava associada a ativi- dades domésticas: faxineiras, empregadas domésticas, cozinheiras e babás. As mulheres que trabalhavam foram agrupadas em dois grandes grupos, segundo o relato das atividades predominantes: $38,2 \%$ referiram maior predomínio de atividades sedentárias (sentadas) e 61,8\% relataram maior atividade física no trabalho (andando ou em movimento). O deslocamento para o trabalho era realizado através de transporte público pela maioria das mulheres (57,6\%). Dessas, 36,4\% relatavam caminhar até o trabalho e $6,1 \%$ não necessitavam deslocar-se para o trabalho.

A atividade física (Tabela 3) não apresentou modificação significativa no decorrer dos períodos, entretanto detecta-se maior gasto energético semanal no grupo Intervenção aos dois anos pós-parto. Ao analisar os domínios que compõem a atividade física total, a mesma diferença pode ser identificada nas atividades domésticas. Contrariamente à atividade física total, é possível identificar aumento no

Tabela 1

Distribuição do número e proporção de mulheres, segundo características socioeconômicas, comportamentais e antropométricas. São Paulo, 2005-2009.

\begin{tabular}{|c|c|c|c|c|c|}
\hline \multirow[t]{2}{*}{ Variáveis } & \multicolumn{2}{|c|}{ Controle } & \multicolumn{2}{|c|}{ Intervenção } & \multirow[t]{2}{*}{ p\# } \\
\hline & $\mathrm{n}$ & $\%$ & $\mathrm{n}$ & $\%$ & \\
\hline Cor da pele & & & & & 0,28 \\
\hline Branca & 13 & 44,8 & 10 & 37,7 & \\
\hline Negra & 5 & 17,2 & 10 & 35,7 & \\
\hline Parda & 11 & 37,9 & 8 & 28,5 & \\
\hline Paridade & & & & & 0,87 \\
\hline Primípara & 12 & 41,3 & 11 & 39,2 & \\
\hline Multípara & 17 & 58,6 & 17 & 60,7 & \\
\hline Escolaridade (anos)* & & & & & 0,22 \\
\hline$<8$ & 11 & 40,7 & 10 & 35,7 & \\
\hline $8-10$ & 8 & 29,6 & 4 & 14,2 & \\
\hline$\geq 11$ & 8 & 29,6 & 14 & 50,0 & \\
\hline Tabagismo & & & & & 0,36 \\
\hline Não fuma/Parou & 27 & 93,1 & 22 & 81,4 & \\
\hline Fuma & 2 & 6,9 & 5 & 18,5 & \\
\hline \multicolumn{6}{|c|}{ Presença de companheiro } \\
\hline Sim & 28 & 96,5 & 25 & 89,2 & 0,58 \\
\hline Não & 1 & 3,4 & 3 & 10,7 & \\
\hline \multicolumn{6}{|c|}{ Estado nutricional pré-gestacional* } \\
\hline Adequado & 16 & 57,1 & 14 & 56,0 & 0,49 \\
\hline Sobrepeso & 11 & 39,2 & 8 & 32,0 & \\
\hline Obesidade & 1 & 3,5 & 3 & 12,0 & \\
\hline
\end{tabular}

\# Teste qui-quadrado; *Houve perda de informações para escolaridade $(n=2)$, tabagismo $(n=1)$ e índice de massa corporal pré-gestacional $(n=3)$. 
Distribuição de mulheres no início da gestação, aos seis e doze meses após o parto, segundo características socioeconômicas e antropométricas. São Paulo, 2005-2009.

\begin{tabular}{|c|c|c|c|}
\hline \multirow[t]{2}{*}{ Variáveis } & Controle & Intervenção & \multirow[t]{2}{*}{$p \#$} \\
\hline & $\bar{X} \pm D P$ & $\bar{X} \pm D P$ & \\
\hline \multicolumn{4}{|c|}{ Início da gestação - $1^{\text {a }}$ consulta } \\
\hline Idade (anos) & $27,93 \pm 6,17$ & $28,72 \pm 4,87$ & 0,59 \\
\hline Renda per capita $(\mathrm{R} \$)^{*}$ & $349,91 \pm 188,23$ & $359,49 \pm 183,05$ & 0,86 \\
\hline Estatura $(\mathrm{cm})$ & $157,42 \pm 5,40$ & $160,41 \pm 6,47$ & 0,06 \\
\hline Peso $(\mathrm{kg})$ & $60,56 \pm 9,73$ & $65,58 \pm 13,54$ & 0,12 \\
\hline IMC (kg/m²) & $24,39 \pm 3,43$ & $25,35 \pm 5,62$ & 0,48 \\
\hline IG (semanas) & $11,64 \pm 3,19$ & $11,35 \pm 3,07$ & 0,74 \\
\hline \multicolumn{4}{|l|}{ Pós-parto } \\
\hline \multicolumn{4}{|l|}{6 meses } \\
\hline Aleitamento (dias) & $103,41 \pm 46,51$ & $100,39 \pm 42,8$ & 0,80 \\
\hline Peso $(k g)$ & $59,44 \pm 10,93$ & $65,76 \pm 14,82$ & 0,72 \\
\hline IMC (kg/m²) & $24,08 \pm 3,87$ & $25,65 \pm 5,89$ & 0,24 \\
\hline \multicolumn{4}{|l|}{1 ano } \\
\hline Aleitamento (dias) & $226,14 \pm 135,73$ & $261,43 \pm 123,88$ & 0,31 \\
\hline Peso $(\mathrm{kg})$ & $58,87 \pm 10,27$ & $63,76 \pm 14,49$ & 0,15 \\
\hline IMC (kg/m²) & $23,89 \pm 3,61$ & $24,85 \pm 5,67$ & 0,45 \\
\hline
\end{tabular}

\# Teste " $\mathrm{t}$ " de Student; IG= idade gestacional; IMC= índice de massa corporal; * Houve perda de informações para a renda.

decorrer do período nas atividades ocupacionais em ambos os grupos, e de locomoção no grupo Intervenção.

Ambos os grupos apresentaram redução significativa das atividades de lazer, destacando-se o fato de que apenas $30 \%$ das mulheres do grupo Intervenção realizavam atividade física no lazer por pelo menos 120 minutos por semana, contra $10 \%$ do grupo Controle aos seis meses, reduzindo para $18 \mathrm{e}$ $4 \%$, respectivamente dois anos após o parto. Ao considerarmos a realização de pelo menos 150 minutos semanais de atividades físicas no lazer seguindo a recomendação mínima para manutenção da saúde verificamos que dois anos após o parto nenhuma mulher do grupo Controle e apenas $7 \%$ no grupo Intervenção atingiam a recomendação. Adicionalmente, a contribuição média das atividades de lazer no gasto energético semanal foi inferior a $2 \%$ aos seis meses e inferior a $1 \%$ aos dois anos após o parto. A caminhada foi a única atividade física no lazer das mulheres do grupo Controle na última entrevista.

A frequência e as principais barreiras que dificultavam a prática regular de atividade física no lazer nesse período são apresentadas na Tabela $4 \mathrm{e}$ Figura 1. Destacam-se a falta de tempo e o cuidado com os filhos, o trabalho e os afazeres domésticos. Apenas o comodismo foi mais frequente na coorte Intervenção $(p=0,029)$.

Ressalta-se o fato de que $82 \%$ das mulheres do grupo Intervenção recordavam-se das orientações nutricionais detalhadamente e apenas $50 \%$ das mulheres lembravam-se das recomendações para a prática regular de atividade física sendo a diferença estatisticamente significativa $(p=0,024)$. Dessas mulheres, $21,4 \%$ seguiram as recomendações até dois anos após o parto, 21,4\% seguiram durante a gestação, 14,3\% durante um período após o parto e $42,9 \%$ relataram não segui-las. No grupo Controle, uma mulher relatou ter recebido orientações para a prática de atividade física e três mulheres orientações sobre alimentação por profissionais de saúde. 
Tabela 3

Mediana e percentis do gasto energético (MET-hora/semana) nos três momentos nos grupos Intervenção e Controle. São Paulo, 2005-2009.

\begin{tabular}{|c|c|c|c|c|c|}
\hline \multirow[t]{2}{*}{ Variável } & \multicolumn{2}{|c|}{ Controle } & \multicolumn{2}{|c|}{ Intervenção } & \multirow[t]{2}{*}{$p^{*}$} \\
\hline & Mediana & $\mathrm{P}_{25} ; \mathrm{P}_{75}$ & Mediana & $P_{25} ; P_{75}$ & \\
\hline \multicolumn{6}{|c|}{ Gasto energético semanal } \\
\hline Seis meses & 247,80 & 182,$34 ; 288,15$ & 279,51 & 220,$01 ; 329,99$ & 0,27 \\
\hline Um ano & 264,93 & 194,$86 ; 341,67$ & 253,64 & 199,$86 ; 329,90$ & 0,62 \\
\hline \multirow[t]{2}{*}{ Dois anos } & 238,94 & 187,$58 ; 325,94$ & 329,50 & 247,$74 ; 407,04$ & 0,007 \\
\hline & & $0,381 \S$ & & $0,174 \S$ & \\
\hline \multicolumn{6}{|c|}{ Atividade doméstica } \\
\hline Seis meses & 163,80 & 127,$93 ; 239,40$ & 198,98 & 147,$53 ; 260,75$ & 0,31 \\
\hline Um ano & 202,65 & 124,$43 ; 266,18$ & 199,15 & 146,$30 ; 243,08$ & 0,63 \\
\hline \multirow[t]{2}{*}{ Dois anos } & 155,58 & 117,$34 ; 184,54$ & 272,30 & 169,$40 ; 305,55$ & 0,001 \\
\hline & & $0,131 \S$ & & $0,404 \S$ & \\
\hline \multicolumn{6}{|c|}{ Atividade ocupacional } \\
\hline Seis meses & 0 & $0 ; 10,05$ & 0 & $0 ; 4,46$ & 0,50 \\
\hline Um ano & 0 & $0 ; 25,55$ & 0 & $0 ; 34,13$ & 0,45 \\
\hline \multirow[t]{2}{*}{ Dois anos } & 9,28 & $0 ; 100,10$ & 18,55 & $0 ; 70,00$ & 0,70 \\
\hline & & $0,039 \S$ & & $0,060 \S$ & \\
\hline \multicolumn{6}{|c|}{ Atividade locomoção } \\
\hline Seis meses & 26,23 & 18,$44 ; 82,25$ & 30,62 & 21,$55 ; 30,62$ & 0,73 \\
\hline Um ano & 24,93 & 15,$29 ; 93,53$ & 22,08 & 14,$38 ; 22,08$ & 0,49 \\
\hline \multirow[t]{2}{*}{ Dois anos } & 45,32 & 15,$08 ; 115,83$ & 47,43 & 22,$99 ; 100,80$ & 0,85 \\
\hline & & $0,371 \S$ & & $0,032 \S$ & \\
\hline \multicolumn{6}{|c|}{ Atividade lazer } \\
\hline Seis meses & 0 & $0 ; 0,57$ & 1,20 & $0 ; 9,88$ & 0,02 \\
\hline Um ano & 1,50 & $0 ; 7,70$ & 0 & $0 ; 5,15$ & 0,18 \\
\hline \multirow[t]{2}{*}{ Dois anos } & 0 & $0 ; 0$ & 0 & $0 ; 1,61$ & 0,18 \\
\hline & & $0,003 \S$ & & $0,040 \S$ & \\
\hline
\end{tabular}

* Teste "U" de Mann-Whitney para comparação entre os grupos; § Teste de Friedman para amostras dependentes.

\section{Tabela 4}

Distribuição do número e proporção de mulheres, segundo as barreiras relatadas para a prática de atividade física no pós-parto. São Paulo, 2005-2009.

\begin{tabular}{|c|c|c|c|c|c|}
\hline \multirow[t]{2}{*}{ Barreiras } & \multicolumn{2}{|c|}{ Controle } & \multicolumn{2}{|c|}{ Intervenção } & \multirow[t]{2}{*}{$p \#$} \\
\hline & $\mathrm{n}$ & $\%$ & $\mathrm{n}$ & $\%$ & \\
\hline 0 & 0 & - & 3 & 10,7 & 0,132 \\
\hline 1 & 15 & 51,7 & 9 & 32,1 & \\
\hline 2 & 8 & 27,6 & 11 & 39,3 & \\
\hline 3 & 4 & 13,8 & 5 & 17,9 & \\
\hline 4 & 2 & 0,7 & 0 & - & \\
\hline
\end{tabular}

\# Teste qui-quadrado. Barreiras: falta de tempo, dinheiro e companhia, cuidado com os filhos, afazeres domésticos e casamento, trabalho, comodismo e violência. 
Distribuição da proporção de barreiras relatadas para a prática da atividade física nas duas coortes estudadas. São Paulo, 2005-2009.

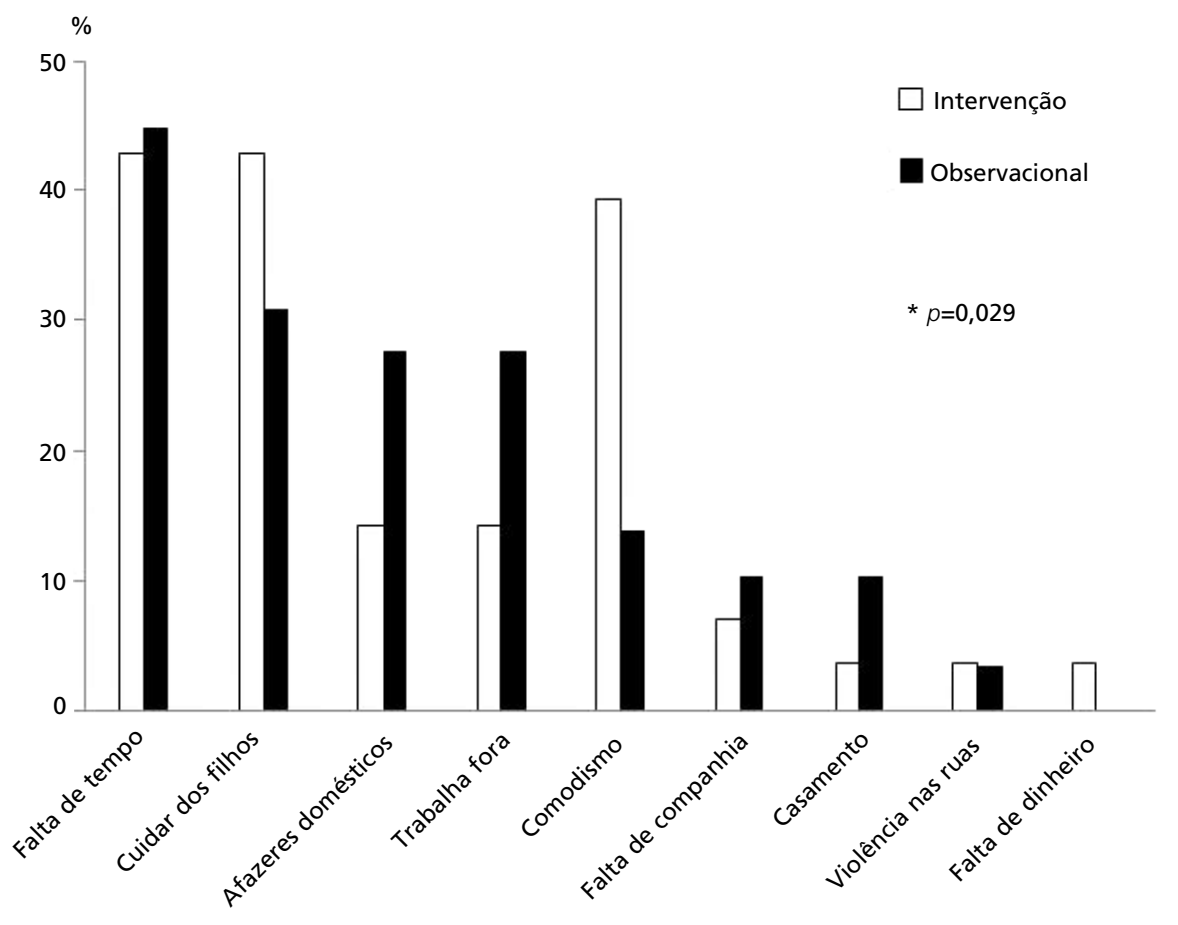

* Teste qui-quadrado.

\section{Discussão}

Buscou-se manter a simplicidade da mensagem da atividade física mínima para a saúde "a realização de pelo menos 30 minutos de caminhada moderada por pelo menos cinco dias na semana", no entanto a prática de atividade física no lazer é pouco representativa resultando em uma parcela inferior ao padrão populacional $20 \mathrm{em}$ ambos os grupos aos dois anos pós-parto. Como observado as mulheres que receberam orientações apresentaram maior gasto energético semanal, no entanto este gasto é predominantemente das atividades domésticas. Assim como, Hinton e Olson 21 identificaram que apenas $15 \%$ de cerca de 500 mulheres exercitavam-se frequentemente um ano após o parto, dentre as atividades, destacavam-se os afazeres domésticos pesados e a caminhada rápida. Como observado em nosso estudo houve maior contribuição das atividades domésticas para o gasto energético. Três ensaios clínicos randomizados também não encontraram melhoria na prática regular de atividade física e na alimentação com metodologias semelhantes. 12,22,23 Kinnunen et al.,22 também utilizando aconselhamento individual, não encontraram diferença significativa na atividade física de lazer dez meses após o parto, apenas aumento da porção de fibras na dieta. Ostbye et al.,23 acompanhando mulheres com sobrepeso e obesidade anterior à gravidez, também não encontraram mudanças comportamentais positivas na alimentação e atividade física dois anos após o parto.

A adoção de um estilo de vida ativo tem sido estimulada e diversas estratégias experimentadas. Chang et al. 12 com o programa piloto Mothers in Motion, através de materiais educativos e aconselhamento em grupo, identificaram que menos da metade do grupo de Intervenção participou das sessões em grupo e assistiu ao material audiovisual, evidenciando dessa maneira a dificuldade em manter as gestantes em programas de orientação. Outro ensaio clínico randomizado, 13 incluía também feedback personalizado com nutricionista e a utilização de pedômetro. Entretanto, contrariamente ao esperado, um ano após o parto, identificou-se que as mulheres que mantiveram a prática de atividade física no lazer tinham maior escolaridade, menor IMC e não participaram das sessões de exercício físico supervisionado oferecidas a todas as mulheres 
do grupo de intervenção. A orientação personalizada com nutricionistas durante a gestação até um ano após o parto, além de envio de folhetos informativos pelo correio, também não foram suficientes para a realização regular de caminhada.

Ainda que programas de intervenção visem à promoção da saúde e à redução do risco de doenças, aumentando o bem-estar de indivíduos e comunidade, poucos têm resultados satisfatórios. A promoção de hábitos saudáveis no período gestacional tem sido indicada devido ao momento propício a mudanças de comportamento. Em recente revisão sistemática de literatura Skouteris et al. ${ }^{24}$ identificaram, em estudos de intervenção durante a gestação, que quatro dentre dez estudos obtiveram resultados positivos em relação ao comportamento alimentar e apenas um relativo à atividade física.

A compreensão de possíveis fatores que dificultam a prática regular de atividade física tem sido investigada através das barreiras relatadas pelas mulheres. Como em diversos estudos, $23,25,26$ a falta de tempo e o cuidado com os filhos foram as barreiras mais citadas em ambos os grupos para explicar a não realização de atividade física regular. Destaca-se, ainda na amostra estudada, o comodismo, um fator pessoal que pode desmotivar a prática de atividade física, ter sido significativamente mais citado pelo grupo Intervenção. Outros estudos detectaram o relato de cansaço das mães, 25 problemas como insegurança, falta de transporte e dinheiro. 21 Carter-Edwards et al.26 identificaram nas mulheres que não participaram do programa a prioridade em outras atividades em detrimento da própria saúde, destacando a falta de apoio da rede social. Ainda que no programa Active Mothers Postpartum, as mulheres tenham relatado sentir os benefícios da prática regular da atividade física, o cuidado com $\mathrm{a}(\mathrm{s})$ criança(s) e o trabalho foram indicados como dificuldades a serem superadas. ${ }^{23}$ Ainda que se identifique maior gasto energético das mulheres que receberam orientação, esse era predominantemente das atividades domésticas o que pode indicar reduzida autonomia e desenvoltura para adequar o tempo, o cuidado dos filhos, da casa, do casamento e do trabalho por parte das mulheres para transformar as condições e possibilitar a prática de atividade física no lazer.

Complementando os demais estudos, Chang et $a l ., 27$ analisaram os fatores que motivaram comportamentos saudáveis, sendo relacionados com a aparência física, desconforto físico, e preocupação com o desenvolvimento de doenças relacionadas ao sobrepeso/obesidade, e de rede de apoio social. De modo similar, as barreiras relatadas para a adoção de estilo de vida saudável focaram as necessidades da criança, negligenciando-se os cuidados pessoais. ${ }^{24}$ Adicionalmente, a baixa autoeficácia e autoestima e a falta de autocontrole e conhecimento, principalmente em relação a comportamentos alimentares, são corroborados por Larson-Meyer, 28 em revisão da literatura, na qual identificou que o exercício moderado sem restrição calórica não promove maior perda de peso. Entretanto, mulheres com altos níveis de atividade física no período pós-parto têm maior probabilidade de retornar ao seu peso pré-gestacional e reter menos peso. ${ }^{28}$ No entanto, essa intensidade de atividade física não foi identificada na presente amostra.

Essas barreiras refletem a natureza multidimensional da mudança do ciclo de vida dessas mulheres. $26 \mathrm{O}$ apoio social pode motivar mudanças comportamentais no estilo de vida e a manutenção que devem ser incluídas nas estratégias de programas de intervenção. 23,26

Os sentimentos acerca da maternidade, a autoeficácia para exercitar-se e a intenção de fazer exercícios físicos foram preditores positivos para a prática um ano após o parto. ${ }^{21}$ No presente estudo, a orientação individualizada por nutricionistas treinadas implicava na transmissão da mensagem e não no planejamento conjunto, o que pode ter sido uma limitação. Hilton e Olson ${ }^{21}$ supõem que as mulheres terão maior probabilidade de exercitar-se regularmente se possuírem um plano e estiverem confiantes em sua capacidade para a realização dos exercícios.

Outro fator limitante do estudo refere-se ao fato de que nutricionistas (treinadas) realizavam tanto a orientação nutricional quanto a de atividade física. A forma de orientação e o profissional envolvido devem ser analisados com cautela, uma vez que apenas metade das mulheres da intervenção recordava as orientações relativas à prática de atividade física, porém maior número recordava as orientações nutricionais recebidas. Outro possível viés está relacionado à utilização do peso referido para a classificação do estado nutricional pré-gestacional. Segundo Peixoto et al., ${ }^{29}$ a utilização do peso e estatura referidos pode reduzir a sensibilidade para a classificação do estado nutricional em mulheres com sobrepeso e obesas. Não houve diferença do peso referido em relação ao mensurado na amostra representativa da população no estudo de validação, no entanto os autores encontraram diferença de cerca de $2,2 \mathrm{~cm}$ na altura das mulheres. No presente estudo a estatura não foi referida minimizando o problema encontrado por Peixoto et al.29 Além disso, o peso referido se torna um método de baixo custo e que 
permite avaliar amostras representativas da população. ${ }^{29}$

No período pós-parto, como observado, ocorrem diversas modificações no cotidiano das mulheres, devido a maior necessidade de cuidados com o bebê e consequente mudança do foco para o filho. Fatores como autoestima, apoio social e conhecimento repercutem na adoção de um estilo de vida saudável.13 Em consonância com Farinatti e Ferreira, ${ }^{30}$ concluímos que deve-se pensar em programas que possibilitem a aquisição de conhecimentos conceituais e procedimentais para a prática regular de exercício físico com autonomia. A proposição de programas de intervenção deve

\section{Referências}

1. OMS (Organização Mundial da Saúde). Análise da estratégia global para alimentação, atividade física e saúde. Epidemiol Serv Saúde. 2005; 14: 41-68

2. Matsudo VKR. Physical activity: passport for health. World Health Report. 1997; 50: 16-17.

3. ACSM (American College of Sports Medicine). The recommended quantity and quality of exercise for developing and mantaining cardiorespiratory and muscular fitness, and flexibility in healthy adults. Med Sci Sports Exerc. 1998; 30: $975-91$.

4. Haskell WL, Lee I-M, Pate RP, Powell KE, Blair SN, Franklin BA, Macera CA, Heath GW, Thompson PD, Bauman A. Physical activity and public health: updated recommendation for adults from the American College of Sports Medicine and the American Heart Association. Circulation. 2007; 116: 1081-93.

5. Impact of physical activity during pregnancy and postpartum on chronic disease risk. Med Sci Sports Exerc. 2006; 38: 989-1006

6. ACOG (American College of Obstetricians and Gynecology). Exercise during pregnancy and the postpartum period. Clin Obst Gynecol. 2003; 46: 496-9.

7. WHO (World Health Organization). Obesity: preventing and managing the global epidemic. Report of a WHO consultation on Obesity. Geneva: WHO/NUT/NCD/98.1; 1998: 1-276.

8. Peterson KE, Sorensen G, Pearson M, Hebert JR, Gottlieb BR, McCormick MC. Design of an intervention addressing multiple levels of influence on dietary and activity patterns of low-income, postpartum women. Health Educ Res. 2002; 17: 531-40.

9. Clapp JF, Simonian S, Lopez B, Appleby-Wineberg S, Harcar-Sevcik R. The one-year morphometric and neurodevelopmental outcome of the offspring of women who continued to exercise regularly throughout pregnancy. Am J Obstet Gynecol. 1998; 178: 594-9.

10. Blum JW, Beaudoin CM, Caton-Lemos L. Physical activity patterns and maternal well-being in postpartum women. Matern Child Health J. 2004; 8: 163-9. considerar o ambiente físico e social, tornando-se um facilitador na mudança/manutenção de comportamentos e na superação das barreiras.

A identificação da prática insignificante de atividade física no lazer das mulheres dois anos após o parto induz a reflexão da utilização de recomendação mínima da atividade física para a manutenção da saúde, a qual deve ser planejada adequadamente nas intervenções. Visto que esse é apenas o primeiro passo para a adoção de um estilo de vida ativo sugerimos a necessidade da divulgação de uma mensagem que indique a progressão da intensidade, frequência e duração dos tipos de exercício físico para a melhoria da aptidão física.

11. Krummel D, Semmens E, MacBride AM, Fisher B. Lesson learned from the mothers overweight management study in 4 West Virginia WIC offices. J Nutr Educ Behav. 2010; 42 (3 Suppl.): S52-8.

12. Chang MW, Nitzke S, Brown R. Design and outcomes of a mothers in motion behavioral intervention pilot study. $\mathrm{J}$ Nutr Educ Behav. 2010; 42 (3 Suppl.): S11-21.

13. Krummel DA, Semmens E, Boury J, Gordon PM, Larkin KT. Stages of change for weight management in postpartum women. J Am Diet Ass. 2004; 104: 1102-8.

14. Institute of Medicine. Subcommitee for a Clinical Application Guide, Committee on Nutrition Status during pregnancy and lactation, Food and Nutrition Board, National Academy of Sciences. Nutrition during pregnancy and lactation. An implementation guide. Washington: National Academy Press; 1992.

15. Chasan-Taber L, Schmidt MD, Roberts DE, Hosmer D, Markensonn G, Freedson PS. Development and validation of a pregnancy physical activity questionnaire. Med Sci Sports Exerc. 2004; 36: 1750-60.

16. Ainsworth BE, Haskell WL, Whitt MC, Irwin ML, Swartz AM, Strath SJ, O'Brien WL, Bassett DR Jr, Schmitz KH, Emplaincourt PO, Jacobs DR Jr, Leon AS. Compendium of physical activities: an update of activity codes and MET intensities. Med Sci Sports Exerc. 2000; 32 (9 Suppl.): S498-504.

17. Jellife DB, Jellife EFP. Community nutritional asssessment. New York: Oxford University Press; 1989.

18. Habicht JP. Estandarización de métodos epidemiológicos cuantitativos sobre el terreno. Bol Oficina Sanit Panam. 1974; 76 : 375-84

19. Atalah E, Castillo C, Castro R, Aldea A. Propuesta de um nuevo estándar de evaluación nutricional em embarazadas. Rev Med Chile. 1997; 125: 1429-36.

20. Brasil. Ministério da Saúde. Secretaria de Vigilância em Saúde. Secretaria de Gestão Estratégica e Participativa. Vigitel Brasil 2010: vigilância de fatores de risco e proteção para doenças crônicas por inquérito telefônico / Ministério da Saúde, Secretaria de Vigilância em Saúde, Secretaria de Gestão Estratégica e Participativa. Brasília, DF; 2011. 
21. Hinton PS, Olson CM. Postpartum exercise and food intake: the importance of behavior-specific self-efficacy. $\mathrm{J}$ Am Diet Assoc. 2001; 101: 1430-7.

22. Kinnunen TI, Pasanen M, Aittasalo M, Fogelholm M, Weiderpass E, Luoto R. Reducing postpartum weight retention - a pilot trial in primary health care. Nutr J. 2007; 6: 21.

23. Ostbye T, Krause KM, Lovelady CA, Morey MC, Bastian LA, Peterson BL, Swamy GK, Brouwer RJ, McBride CM. Active mothers postpartum: a randomized controlled weight-loss intervention trial. Am J Prev Med. 2009; 37: 173-80.

24. Skouteris H, Hartley-Clark L, McCabe M, Milgrom J, Kent B, Herring SJ, Gale J. Preventing excessive gestational weight gain: a systematic review of interventions. Obes Rev. 2010; 11: 757-68.

25. Evenson KR, Aytur SA, Borodulin K. Physical activity beliefs, barriers, and enablers among postpartum women. J Womens Health (Larchmt). 2009; 18: 1925-34.
26. Carter-Edwards L, Østbye T, Bastian LA, Yarnall KSH, Krause KM, Simmons TJ. Barriers to adopting a healthy lifestyle: insight from postpartum women. BMC Res Notes. $2009 ; 2: 161-4$

27. Chang M, Nitzke S, Guilford E, Adair CH, Hazard DL. Motivators and barriers to healthful eating and physical activity among low-income overweight and obese mothers. J Am Diet Assoc. 2008; 108: 1023-8.

28. Larson-Meyer DE. Effect of postpartum exercise on mothers and their offspring: a review of the literature. Obes Res. 2002; 10: 841-53.

29. Peixoto M do RG, Benício MHA, Jardim PCBV. Validade do peso e da altura auto-referidos: o estudo de Goiânia. Rev Saúde Pública. 2006; 40: 1065-72.

30. Farinatti P, Ferreira M. Saúde, promoção da saúde e educação física. Rio de Janeiro: Ed. UERJ; 2006.

Recebido em 20 de junho de 2011

Versão final apresentada em 26 de março de 2012

Aprovado em 9 de abril de 2012 